\title{
Fault detection in $\lambda$-tuned control loops using an observer with integral action
}

\author{
Magnus Berndtsson*c and Andreas Johansson*
}

\begin{abstract}
A model-based fault detection algorithm assuming uncertain process parameters is used for detecting poorly operating $\lambda$-tuned control loops. The a priori information obtained from $\lambda$-tuning is used to create an observer as residual generator. The observer has integral action which makes it possible to obtain a tight fault detection threshold. A linear optimization approach is used for finding the parameters in the threshold.
\end{abstract}

\section{INTRODUCTION}

This work focuses on fault detection for a special kind of controller, the $\lambda$-tuned PI controller. The $\lambda$-tuning is a standard developed by the swedish pulp and paper industry [10] [9] for tuning PI controllers for first order systems with time-delay i.e.

$$
G(s)=\frac{e^{-L s} k}{T s+1}
$$

It is a method based on the IMC (Internal Model Control) principle which is easy to apply and is intended to be used by control-room operators. The method has become very popular in the pulp and paper industry because of its simplicity and is now spreading to other industries.

Some examples addressing the problem of detecting and diagnosing oscillations are [4], [11], [8], and [6]. Methods for comparing the controller performance to an optimal controller have also been developed [7].

Instead of concentrating on one problem, like for example the oscillations, the proposed method is based on a general fault detection method. It has therefore the potential to detect different kinds of deviations in the control loop, such as sticking or leaking valves, faulty sensors, and in some cases, bad controller tuning.

The general idea behind model-based fault detection is to use the redundancy in the information obtained from measurements in combination with a process model to decide if a fault has occurred [5]. The prime disadvantage with model-based fault detection is the necessity to have an analytical process model. This is why model-based fault detection is well suited to

\footnotetext{
*Control Engineering Group Luleå University of Technology

SE-971 87 Luleå, Sweden

${ }^{c}$ corresponding author: magnus.berndtsson@1tu.se
}

$\lambda$-tuned control loops where a process model on the form of (1) is obtained during tuning [3].

A fault detection algorithm consists of two steps: the residual generation and the residual evaluation. The residual is a signal which ideally is nonzero when there is a fault and zero otherwise. However, due to disturbances and noise this can not be achieved exactly. The problem in the residual generation is then to find a signal as close to zero as possible when no fault has occurred. One way of creating that kind of residual is to use the residual from a Luenberger observer. The evaluation step may then be designed based on the dynamics of the estimation error [1].

Due to the model uncertainties the residual will be affected by the known input signals. To achieve a threshold that is as tight as possible to the residual, the threshold should also depend on the known input signals. This is accomplished by assuming a process model with time-varying parameters [1].

Because of the model uncertainty the threshold may have to be chosen very large to avoid false alarms. In this work the observer that creates the residual features integral action to make it possible to achieve a tighter threshold and thus detect smaller faults.

\section{PRELIMINARIES}

In the following, $|\cdot|$ denotes element-wise absolute value when applied to a matrix. Inequalities between matrices is also to be interpreted element-wise. The notation $\otimes$ represents the Kronecker product, for which a useful property is

Property 1: Let $x \in \mathbb{R}^{n}$ and $y \in \mathbb{R}^{m}$. Then $x \otimes y=$ $\left(x \otimes I_{m}\right) y=\left(I_{n} \otimes y\right) x$

The pseudoinverse of a matrix $A$ is denoted $A^{+}$. The notation $1_{n}$ represents a column vector of ones of dimension $n$.

A linear operator defined by convolution by a weighting function is denoted by the symbol of the weighting function written in bold-face font, thus e.g.

$$
\mathbf{F} G(t) \triangleq(F * G)(t)=\int_{0}^{t} F(t-\tau) G(\tau) d \tau
$$


For functions $|\cdot|$ is to be interpreted pointwise, so that $|F|(t) \triangleq|F(t)|$. Inequalities between functions is also intended pointwise, i.e. $F \leq G$ means $F(t) \leq G(t)$ for all $t$. For the calculations in the sequel, the following result from [1] is required.

Lemma 1: Let $F(t) \in \mathbb{R}^{n \times m}$ and $G(t)$, $H(t) \in \mathbb{R}^{m \times r}$. If $F \geq 0$ and $H \geq G$ then $F * H \geq F * G$.

\section{FAULT DETECTION ALGORITHM}

Consider the system,

$$
\begin{aligned}
\dot{x} & =f(x, u, \pi)+\mu \\
y & =C x+D \pi+\nu
\end{aligned}
$$

where $x(t) \in \mathbb{R}^{n}$ represents the state vector, $u(t)$ is the input, $y(t) \in \mathbb{R}^{\ell}$ is the output, $\mu(t) \in \mathbb{R}^{n}$ is the process noise, $\nu(t) \in R^{\ell}$ is the measurement noise and $\pi(t) \in \mathbb{R}^{m}$ represents the uncertain parameters. In [1], it is shown that if $f$ is near linear in $x$ and $u$ and $\pi$ is small, a second order Taylor expansion of 2 may be expressed as

$$
\begin{aligned}
\dot{x} & =A x+P(\pi \otimes x)+E \pi+F+\mu \\
y & =C x+D \pi+\nu
\end{aligned}
$$

where

$$
\begin{aligned}
A & \triangleq f_{x}^{\prime}(0,0,0) \in \mathbb{R}^{n \otimes n} \\
P & \triangleq f_{x \pi}^{\prime \prime}(0,0,0) P \in \mathbb{R}^{n \times n m} \\
E(t) & \triangleq f_{\pi}^{\prime}(0,0,0)+f_{u \pi}^{\prime \prime}(0,0,0)(I \otimes u(t)) \in \mathbb{R}^{n \times m} \\
F(t) & \triangleq f_{u}^{\prime}(0,0,0) u(t) \\
& +f_{u u}^{\prime \prime}(0,0,0)(u(t) \otimes u(t)) \in \mathbb{R}^{n}
\end{aligned}
$$

It is assumed that $A$ is Hurwitz.

An observer with integral action has been chosen as the residual generator. The integral action will make it possible to detect fast changes in the process parameters by enabling a tight threshold. The error system of the observer can also be formulated as (3) with the residual $r(t)$ as output and known signals as inputs.

The dynamic threshold generator is a dynamic system whose inputs are the known process inputs and the output is an upper bound for the residual. The following theorem gives the upper bound for $|y(t)|$ in (3) and is therefore instrumental in developing a dynamic threshold generator for (3) [1].

Theorem 1: Assume that $|\pi(t)| \leq \Pi \in \mathbb{R}^{m}$ for all $t \geq$ 0 and let $G(t) \triangleq e^{A t}$. Let $H(t) \in \mathbb{R}^{n \times n}$ be a function that satisfies $H(t) \geq|G(t) P|\left(\Pi \otimes I_{n}\right)$ for all $t \geq 0$ and let $\mathbf{H}$ be the linear operator defined by convolution with $H(t)$. Let $\Gamma \in \mathbb{R}^{n \times n}$ be a function that satisfies $\Gamma(t) \geq$ $|G(t)|$ for all $t \geq 0$ and let $\Gamma$ be the linear operator defined by convolution with $\Gamma$. Then, if $\|\mathbf{H}\|<1$ for some induced operator norm $\|\cdot\|$ then $\left\|(I-\mathbf{H})^{-1}\right\|<\infty$ and

$|y| \leq|C|(I-\mathbf{H})^{-1}(\boldsymbol{\Gamma}(|E| \Pi+|\mu|)+\Gamma|x(0)|)+|D| \Pi+|\nu|$

Theorem 1 makes it possible to develop fault detection algorithms for a wide spectrum of processes with arbitrary dimensions on the state, input and the output vectors. It will now be used for developing an algorithm to detect significant changes in a process controlled by a $\lambda$-tuned controller.

\section{Residual Generator}

A state-space description of (1) is

$$
\begin{aligned}
\dot{x} & =-\frac{1}{T} x+\frac{k}{T} u+\mu \\
y & =x+\nu
\end{aligned}
$$

where $u$ is defined as

$$
u(t) \triangleq w(t-L)
$$

where $w$ is the actual input to the plant. A process disturbance $\mu$ and a measurement disturbance $\nu$ has also been introduced. The parameters $T$ and $k$ are the time constant and static gain, respectively. It is assumed that these parameters are uncertain and time-varying with known nominal value $T_{0}$ and $k_{0}$, respectively, i.e.

$$
\begin{aligned}
T(t) & =T_{0}\left(1+\pi_{1}(t)\right) \\
k(t) & =k_{0}\left(1+\pi_{2}(t)\right)
\end{aligned}
$$

Let $\pi \triangleq\left[\begin{array}{llll}\pi_{1} & \pi_{2} & \dot{\pi}_{1} & \dot{\pi}_{2}\end{array}\right]^{T}$ and define upper bounds for the parameter uncertainties in (5) as

$$
\Pi=\left[\begin{array}{llll}
\Pi_{1} & \Pi_{2} & \Pi_{3} & \Pi_{4}
\end{array}\right]^{T} \geq|\pi(t)|
$$

The process (5) may then be expressed as (2) with $C=1$ and $D=0$ where

$$
f(x, u, \pi)=-\frac{1}{T_{0}\left(1+\pi_{1}\right)} x+\frac{k_{0}\left(1+\pi_{2}\right)}{T_{0}\left(1+\pi_{1}\right)} u
$$

An observer with integral action for (3) is

$$
\begin{aligned}
\dot{\hat{x}} & =A \hat{x}+F u+K(y-\hat{y})+Q \iota \\
i & =L(y-\hat{y}) \\
\hat{y} & =C \hat{x}
\end{aligned}
$$

i.e.

$$
\begin{aligned}
\dot{\hat{x}} & =-\frac{1}{T_{0}} \hat{x}+\frac{k_{0}}{T_{0}} u+K(y-\hat{y})+\iota \\
i & =L(y-\hat{y}) \\
\hat{y} & =\hat{x}
\end{aligned}
$$


The initial conditions for the observer are set to $\hat{x}(0)=y(0)$ and $\iota(0)=0$.

The dynamics of the estimation error $\tilde{x} \triangleq x-\hat{x}$ are

$$
\begin{aligned}
\dot{\tilde{x}} & =-\left(\frac{1}{T_{0}}+K\right) \tilde{x}+\pi_{1} \frac{1}{T_{0}} x+\left(\pi_{2}-\pi_{1}\right) \frac{k_{0}}{T_{0}} u \\
& -\iota+\mu-K \nu \\
& =-\left(\frac{1}{T_{0}}+K\right) \tilde{x}+\xi+\mu-K \nu
\end{aligned}
$$

where $\xi \triangleq \frac{1}{T_{0}} \pi_{1} x+\frac{k_{0}}{T_{0}}\left(\pi_{2}-\pi_{1}\right) u-\iota$ is introduced as a state variable. The reason for this is that $\xi$ can be considered an estimation error since the purpose of the integral term $\iota$ is to cancel the effects of the uncertainty terms $\pi_{1} \frac{1}{T_{0}} x$ and $\left(\pi_{2}-\pi_{1}\right) \frac{k_{0}}{T_{0}} u$ in (7). The dynamics of this estimation error are

$$
\begin{aligned}
\dot{\xi} & =\frac{1}{T_{0}} \pi_{1} \dot{x}+\frac{1}{T_{0}} \dot{\pi}_{1} x+\frac{k_{0}}{T_{0}}\left(\pi_{2}-\pi_{1}\right) \dot{u}-i \\
& +\frac{k_{0}}{T_{0}}\left(\dot{\pi}_{2}-\dot{\pi}_{1}\right) u \\
& =\frac{1}{T_{0}} \pi_{1}\left(-\frac{1}{T_{0}} x+\frac{k_{0}}{T_{0}} u+\frac{1}{T_{0}} \pi_{1} x+\left(\pi_{2}-\pi_{1}\right) \frac{k_{0}}{T_{0}} u\right. \\
& +\mu)+\frac{1}{T_{0}} \dot{\pi}_{1} x+\frac{k_{0}}{T_{0}}\left(\pi_{2}-\pi_{1}\right) \dot{u}+\frac{k_{0}}{T_{0}}\left(\dot{\pi}_{2}-\dot{\pi}_{1}\right) u \\
& -L(y-\hat{y}) \\
& =\pi_{1} \frac{1}{T_{0}} \xi+\pi_{1}\left(\frac{1}{T_{0}}\left(-\frac{1}{T_{0}} y+\frac{k_{0}}{T_{0}} u+\iota\right)-\frac{k_{0}}{T_{0}} \dot{u}\right) \\
& +\pi_{2} \frac{k_{0}}{T_{0}} \dot{u}-L \tilde{x}+\dot{\pi}_{1}\left(\frac{1}{T_{0}} x-\frac{k_{0}}{T_{0}} u\right)+\dot{\pi}_{2} \frac{k_{0}}{T_{0}} u \\
& +\pi_{1} \frac{1}{T_{0}^{2}}\left(T_{0} \mu-\nu\right)-L \nu
\end{aligned}
$$

With the augmented state vector $z \triangleq\left[\begin{array}{ll}\xi & \tilde{x}\end{array}\right]^{T}$ and the definitions

$$
\begin{gathered}
A_{e} \triangleq\left[\begin{array}{ccc}
0 & -L \\
1 & A-K C
\end{array}\right] \\
P_{e} \triangleq\left[\begin{array}{cccccccc}
\frac{1}{T_{0}} & 0 & 0 & 0 & 0 & 0 & 0 & 0 \\
0 & 0 & 0 & 0 & 0 & 0 & 0 & 0
\end{array}\right] \\
B_{1} \triangleq\left[\begin{array}{l}
1 \\
0
\end{array}\right], B_{2} \triangleq\left[\begin{array}{l}
0 \\
1
\end{array}\right], C_{e} \triangleq\left[\begin{array}{l}
1 \\
0
\end{array}\right]^{T}
\end{gathered}
$$

$$
\begin{aligned}
v_{1} & \triangleq-\frac{1}{T_{0}}\left(-\frac{1}{T_{0}} y+\frac{k_{0}}{T_{0}} u+\iota\right)+\frac{k_{0}}{T_{0}} \dot{u} \\
v_{2} & \triangleq \frac{k_{0}}{T_{0}} \dot{u} \\
v_{3} & \triangleq \frac{1}{T_{0}} x-\frac{k_{0}}{T_{0}} u \\
v_{4} & \triangleq \frac{k_{0}}{T_{0}} u
\end{aligned}
$$

$$
\begin{aligned}
& E_{e} \triangleq\left[\begin{array}{llll}
B_{1} v_{1} & B_{1} v_{2} & B_{1} v_{3} & B_{1} v_{4}
\end{array}\right] \\
& \mu_{e} \triangleq B_{1}\left(\frac{\pi_{1}}{T_{0}} \mu-\frac{\pi_{1}}{T_{0}^{2}} \nu-L \nu\right)+B_{2}(\mu-K \nu) \\
& \nu_{e} \triangleq \nu
\end{aligned}
$$

and $D_{e}=0$ the dynamics of the observer residual can be expressed as

$$
\begin{aligned}
\dot{z} & =A_{e} z+P_{e}(\pi \otimes z)+E_{e} \pi+\mu_{e} \\
r & =C_{e} z+D_{e} \pi+\nu_{e}
\end{aligned}
$$

i.e. the same structure as (3).

\section{Residual Evaluator}

Define $\Gamma(t) \triangleq C_{\Gamma} e^{A_{\Gamma} t} B_{\Gamma} \geq|G(t)|$ and $H \geq$ $\left|G P_{e}\right|\left(\Pi \otimes I_{2}\right)$, where $G(t) \triangleq e^{A_{e} t}$. Let $\boldsymbol{\Gamma}$ and $\mathbf{H}$ be the corresponding linear operators. By choosing $L$ positive and $\left(\frac{1}{T_{0}}+K\right)^{2}<4 L$, complex eigenvalues of $A_{e}$ are avoided which will simplify the calculations of the threshold. Let $\epsilon_{\mu}$ and $\epsilon_{\nu}$ be the upper bounds of the disturbances $\mu$ and $\nu$ defined as $\epsilon_{\mu} \geq\|\mu\|_{\infty}$ and $\epsilon_{\nu} \geq\|\nu\|_{\infty}$, i.e. upper bounds for the infinity norm of the disturbances.

According to Theorem 1, an upper bound for the residual is

$$
\begin{aligned}
|r| & \leq\left|C_{e}\right|(I-\mathbf{H})^{-1}\left(\boldsymbol{\Gamma}\left(\left|E_{e}\right| \Pi+\left|\mu_{e}\right|\right)+\Gamma|z(0)|\right)+|\nu| \\
& =\left|C_{e}\right|(I-\mathbf{H})^{-1}\left(\boldsymbol { \Gamma } B _ { 1 } \left(\left|v_{1}\right| \Pi_{1}+\left|v_{2}\right| \Pi_{2}\right.\right. \\
& \left.+\left|v_{3}\right| \Pi_{3}+\left|v_{4}\right| \Pi_{4}+\left|\frac{\pi_{1}}{T_{0}} \mu-\frac{\pi_{1}}{T_{0}^{2}} \nu-L \nu\right|\right) \\
& +\boldsymbol{\Gamma} B_{2}|\mu-K \nu|+|\nu| \\
& =\mathbf{h}_{\mathbf{1}}\left(\left|v_{1}\right| \Pi_{1}+\left|v_{2}\right| \Pi_{2}+\left|v_{3}\right| \Pi_{3}+\left|v_{4}\right| \Pi_{4}\right. \\
& \left.+\left|\frac{\pi_{1}}{T_{0}} \mu-\frac{\pi_{1}}{T_{0}^{2}} \nu-L \nu\right|\right)+\mathbf{h}_{\mathbf{2}}|\mu-K \nu| \\
& +|\nu| \\
& \leq \mathbf{h}_{\mathbf{1}}\left(\left|v_{1}\right| \Pi_{1}+\left|v_{2}\right| \Pi_{2}+\left|v_{3}\right| \Pi_{3}+\left|v_{4}\right| \Pi_{4}\right) \\
& +\epsilon_{\mu} \frac{\Pi_{1}}{T_{0}} \mathbf{h}_{\mathbf{1}} \theta+\epsilon_{\nu} \frac{\Pi_{1}}{T_{0}^{2}} \mathbf{h}_{\mathbf{1}} \theta+\epsilon_{\nu} L \mathbf{h}_{\mathbf{1}} \theta+\epsilon_{\mu} \mathbf{h}_{\mathbf{2}} \theta \\
& +\epsilon_{\nu} K \mathbf{h}_{\mathbf{2}} \theta=\sigma
\end{aligned}
$$




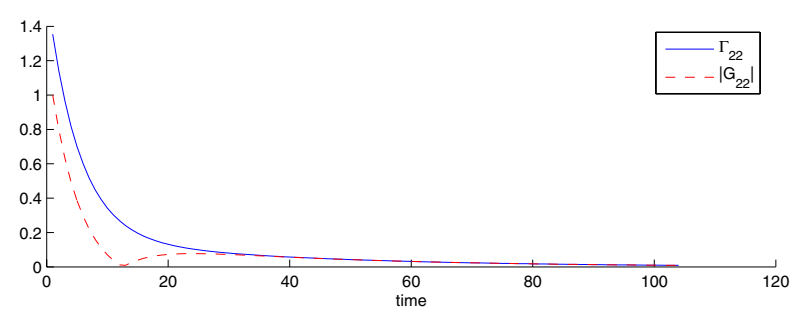

Fig. 1. The modulus of the impulse response $\left|G_{22}(t)\right|$ and its upper bound $\Gamma_{22}(t)$ when $\alpha=0.575$ and $\beta=0.425$

where $h_{1} \triangleq\left|C_{e}\right|(I-\mathbf{H})^{-1} \Gamma B_{1}, h_{2} \triangleq\left|C_{e}\right|(I-$ $\mathbf{H})^{-1} \Gamma B_{2}, \mathbf{h}_{\mathbf{1}}, \mathbf{h}_{\mathbf{2}}$ are the corresponding operators and $\theta$ represents the Heaviside step function. The initial state vector $z(0)$ has been neglected since we only consider the case when the contribution from $z(0)$ has converged to zero. In the last inequality above, Lemma 1 was used.

To find expressions for $h_{1}$ and $h_{2}$ the upper bounds $\Gamma \geq|G|$ and $H \geq\left|G P_{e}\right|(\Pi \otimes I)$ are required. Due to the structure of $P_{e}$, it follows that $\left|G P_{e}\right|=|G| P_{e}$. Thus one may choose $H=\Gamma P_{e}(\Pi \otimes I)$ and the problem reduces to finding upper bounds $\Gamma_{i j}$ for the elements $G_{i j}$. In the Laplace domain, $G$ is

$$
\begin{aligned}
(\mathcal{L} G)(s) & =\left(s I-A_{e}\right)^{-1} \\
& =\frac{1}{s^{2}+2 s \alpha+L}\left[\begin{array}{cc}
(s+2 \alpha) & -L \\
1 & s
\end{array}\right]
\end{aligned}
$$

where $\alpha=\left(\frac{1}{T_{0}}+K\right) / 2$.

Considering the assumptions that $A_{e}$ is Hurwitz and has real eigenvalues it follows that $L>0$ and $\left(\frac{1}{T_{0}}+\right.$ $K)^{2}<4 L$. It may then be shown that $G_{11}, G_{21}$ and $-G_{12}$ are all nonnegative. It is thus possible to choose $\Gamma_{11}=G_{11}, \Gamma_{21}=G_{21}$ and $\Gamma_{12}=-G_{12}$.

Some manipulations yield

$$
G_{22}(t)=\frac{\alpha-\beta}{2 \beta} e^{(\beta-\alpha) t}\left(1-\frac{\alpha+\beta}{\alpha-\beta} e^{-2 \beta t}\right)
$$

where $\beta=\sqrt{\alpha^{2}-L}$. Since $\alpha \geq \beta \geq 0$, an upper bound for $\left|G_{22}\right|$ is

$$
\left|G_{22}(t)\right|=\frac{\alpha-\beta}{2 \beta} e^{(\beta-\alpha) t}\left(1+\frac{\alpha+\beta}{\alpha-\beta} e^{-2 \beta t}\right) \triangleq \Gamma_{22}(t)
$$

The modulus of the impulse response $\left|G_{22}(t)\right|$ and its upper bound $\Gamma_{22}(t)$ is shown in fig. 1.

The Laplace transform of $\Gamma_{22}(t)$ and the other $\Gamma_{i j}(t)$ yields

$$
(\mathcal{L} \Gamma)(s)=\frac{1}{s^{2}+2 s \alpha+L}\left[\begin{array}{cc}
(s+2 \alpha) & -L \\
1 & \frac{\alpha s+L}{\beta}
\end{array}\right]
$$

A drawback with the proposed method is that the resulting threshold (10) requires differentiation of the input due to the terms $\left|v_{1}\right| \Pi_{1}$ and $\left|v_{2}\right| \Pi_{2}$, which may cause problems with unfiltered noisy data. This could be solved by using a simple low pass filter on the inputs to the residual and the threshold. The error introduced by doing so can be significantly reduced by applying the same filter to the measured output also.

\section{THRESHOLD PARAMETER DESIGN}

The upper bound $\Pi$ of the uncertainty vector $\pi$ is unknown. One way to solve this problem is a method that uses stability margins for choosing the upper bounds, suggested in [3]. There, the upper bound $\Pi$ is chosen so that a fault is detected when the parameter changes cause a loss in phase margin that exceeds a preset bound. It is thereby possible to obtain robustness in the supervised control loop.

In this section, an automatic way to determine the unknown upper bounds is suggested using an optimization approach. The criterion on $\Pi$ is that it should be chosen so that, for a set of data with no fault, the threshold always will be larger than the residual. The criterion is possible to express as an optimization problem whose solution gives the parameters.

The upper bound $\Pi$ is substituted by the parameter vector $\pi^{*}$ and the function $H$ consequently by $H^{*}(t)=$ $\Gamma(t) P_{e}\left(\pi^{*} \otimes I_{n}\right)=C_{\Gamma} e^{A_{\Gamma} t} B_{\Gamma} P_{e}\left(\pi^{*} \otimes I_{n}\right)$. The resulting threshold $\sigma$ is, when neglecting the initial state $z(0)$

$$
\sigma \geq\left|C_{e}\right|\left(I-\mathbf{H}^{*}\right)^{-1} \boldsymbol{\Gamma}\left|E_{e}\right| \pi^{*}+|D| \pi^{*}
$$

It is then possible to determine the parameter vector $\pi^{*}$ such that $\sigma \geq|r|$ by solving a linear inequality problem, as shown in [2].

Theorem 2: Let the residual $r$ be generated by the error system (3) with $F=0$ and assume that $|P|\left(I_{m} \otimes\right.$ $\left.|C|^{+}|D|\right)=0$. Let

$$
\begin{aligned}
\Omega & \triangleq \Gamma|E|+|C|^{+}|D|+\boldsymbol{\Gamma}\left(|P|\left(I_{m} \otimes|C|^{+}|r|\right)\right. \\
\varphi & \triangleq|C|^{+}|r|
\end{aligned}
$$

and choose $\pi^{*}$ such that

$$
\Omega(t) \pi^{*}-\varphi(t) \geq 0, \forall t
$$

then

$$
\sigma(t) \geq|r(t)|, \forall t
$$

For details and Proof see [2].

When the vector $\pi^{*}$ is to be determined, using Theorem 2, measurements without faults are required. The sampling interval of the measurements is denoted $h$ and $M$ is the total number of samples. Since it is also desirable that $\left(I-\mathbf{H}^{*}\right)^{-1}$ is stable, the set of admissible 
parameters is defined as $\mathrm{D}=\left\{\pi^{*} \geq 0 \mid \Sigma \pi^{*} \geq \varsigma,(I-\right.$ $\left.\mathbf{H}^{*}\right)^{-1}$ is stable $\}$ where

$$
\begin{aligned}
\Sigma & =\left[\begin{array}{llll}
\Omega(h)^{T} & \Omega(2 h)^{T} & \ldots & \Omega(M h)^{T}
\end{array}\right] \\
\varsigma & =\left[\begin{array}{llll}
\varphi(h)^{T} & \varphi(2 h)^{T} & \ldots & \varphi(M h)^{T}
\end{array}\right]
\end{aligned}
$$

In order to express a condition for stability of $(I-$ $\left.\mathbf{H}^{*}\right)^{-1}$ as a linear inequality, the following Lemma from [2] will be used

Lemma 2: Let $Q(t)=C e^{A t} B \geq 0$. Then $(I-\mathbf{Q})^{-1}$ is stable if $-C A^{-1} B 1_{n} \leq 1_{n}$.

It thus follows that $\left(I-\mathbf{H}^{*}\right)^{-1}$ is stable if $-C_{\Gamma} A_{\Gamma}^{-1} B_{\Gamma} P_{e}\left(\pi^{*} \otimes I_{n}\right) 1_{n} \leq 1_{n}$. which, using Property 1 , can be rewritten as $-C_{\Gamma} A_{\Gamma}^{-1} B_{\Gamma} P_{e}\left(I_{m} \otimes 1_{n}\right) \pi^{*} \leq 1_{n}$ which is a linear inequality in $\pi^{*}$.

An optimal choice of $\pi^{*}$ can be found by minimizing $\Omega \pi^{*}-\varphi$ with respect to some norm. For the 1-norm this is equivalent to minimizing $\sum_{i=1}^{M n} \Sigma_{i} \pi^{*}$ where $\Sigma_{i}$ is the $i$ :th row of $\Sigma$. The linear optimization problem to find $\pi^{*}$ can thus be stated as

$$
\left\{\begin{array}{c}
\min \\
\Sigma^{*} \pi^{*} \leq \varsigma^{*} \\
\pi^{*} \geq 0
\end{array} \quad \Psi \pi^{*}\right.
$$

where

$$
\begin{aligned}
\Psi & =1_{M n}^{T} \Sigma, \varsigma^{*}=\left[\begin{array}{cc}
\varsigma^{T} & 1_{n}^{T}
\end{array}\right]^{T} \\
\Sigma^{*} & =\left[\begin{array}{ll}
\Sigma^{T} & \left(C_{\Gamma} A_{\Gamma}^{-1} B_{\Gamma}\left|P_{e}\right|\left(I_{m} \otimes 1_{n}\right)\right)^{T}
\end{array}\right]^{T}
\end{aligned}
$$

For (8) the functions $\varphi$ and $\Omega$ in Theorem 2 are

$$
\begin{aligned}
\Omega & =\Gamma\left|E_{e}\right|+\Gamma\left|P_{e}\right|\left(I_{4} \otimes\left|C_{e}\right|^{+}|r|\right) \\
\varphi & =\left|C_{e}\right|^{+}|r|
\end{aligned}
$$

where $C_{e}^{+}=\left[\begin{array}{cc}1 & 0\end{array}\right]^{T}$. We see that $D_{e}=0$ implies that $\left|P_{e}\right|\left(I_{m} \otimes\left|C_{e}\right|^{+}\left|D_{e}\right|\right)=0$ which is a prerequisite for Theorem 2.

\section{EXPERIMENTAL SETUP}

The experiments are done in a laboratory environment on a small physical water tank system. The system can be described as

$$
i=-\frac{a \sqrt{2 g l}}{A}+\frac{1}{A} u
$$

where $l$ is the water level, $a$ is the size of the hole through which the outflow takes place, $A$ is the crosssection area of the water tank and $u$ is the control signal to the pump that feeds the water into the tank.
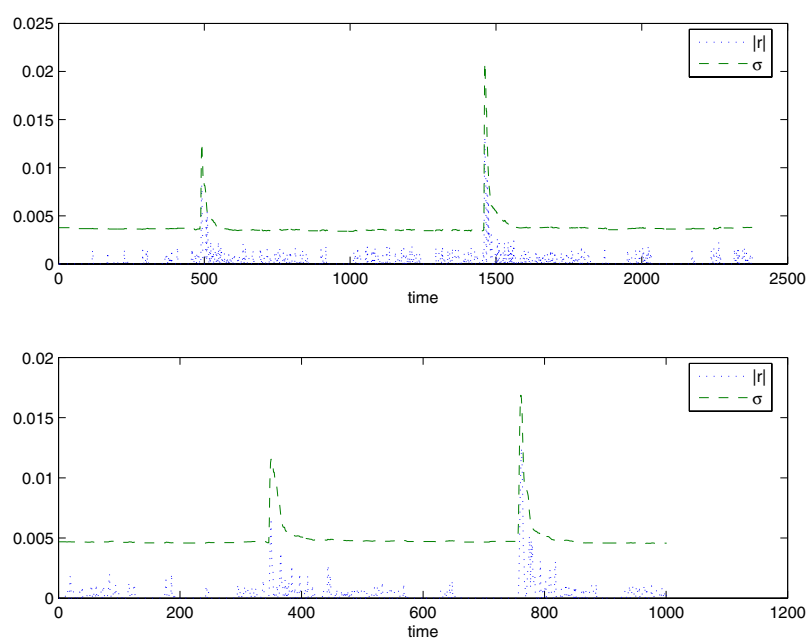

Fig. 2. Design of upper bounds for uncertainties (upper figure) and verification (lower figure)

A step-response experiment has been performed on the water tank and the transfer function was identified to be

$$
G(s)=\frac{2 e^{-2 s}}{1+38 s}
$$

The dead-time $e^{-2 s}$ is probably some of the dynamics of the pump in combination with some delay in the D/A converter.

The controller was $\lambda$-tuned and the controller parameters were set to $\lambda=38, T_{i}=38$ and $k_{c}=\frac{19}{40}$.

\section{EXPERIMENTAL RESULTS}

The upper bounds of the uncertainties in the experiments have been designed with the method described in section "Threshold parameter design". The upper bounds of the measurement and process noises have been determined manually to get a threshold tight to the residual. In these experiments they are set to $\epsilon_{\nu}=\epsilon_{\mu}=0.001$.

The results of the optimization are shown in fig 2 . The upper figure shows the identification on a set of measurement data with no fault. The parameters are here determined using (11) to make the threshold larger than the residual for all time. The lower figure shows a validation on a different set of measurement data and it shows that there are no false alarms when using the parameters obtained from the identification data set. The obtained parameter vector $\pi^{*}$ is $\left[\begin{array}{llll}0.4 & 0.4 & 0 & 0.001\end{array}\right]^{T}$.

Fig 3 shows a comparison between the observer with integral action (lower figure) and the observer without integral action (upper figure) as described in [3]. It is the nonlinearity contribution to the threshold that 

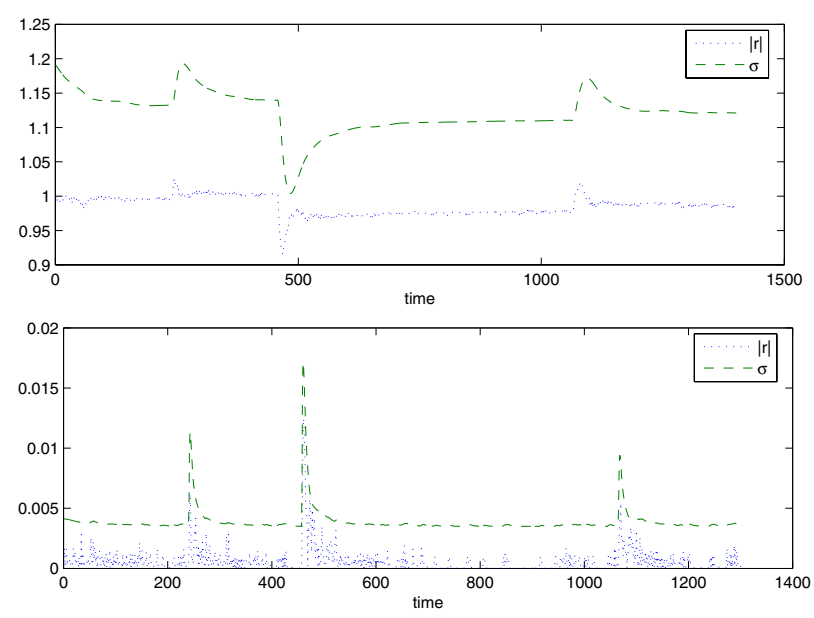

Fig. 3. A comparison between using an observer with integral action (lower figure) and an observer without integral action (upper figure).

is canceled with the integral action and thereby the threshold gets tighter to the residual when using integral action. There are step changes in the reference signal at times 230, 480 and 1100. The step changes give step contributions to the residual and the threshold in the case without integral action and transient contributions in the case with integral action.

The residual with integral action gets smaller because the integral term in the observer cancels the effect of nonlinearities in the process. That makes it possible to make the threshold tighter and thereby get more reliable detections. Figure 4 shows that it is possible to detect smaller faults with integral action in the residual generator without increasing the risk of false alarms. Just before time 800 the output of the water tank is widened to simulate a change in the process parameters. The fault is detected in the case with integral action (lower figure) but in the case without integral action (upper figure) the threshold stays above the residual.

\section{CONCLUSIONS AND FUTURE WORK}

The method presented detects faults in $\lambda$-tuned control loops. As an example it is shown that it detects the widening of the output of an experimental water tank setup. It is also demonstrated how the fault detection threshold follows the behavior of the residual when there are step changes in the residual.

We see from the experimental results that the observer with integral action makes the residual smaller and the threshold tighter compared to an observer without integral action. This makes it possible to detect smaller faults without increased risk of false alarms. An automatic method for finding the required parameters
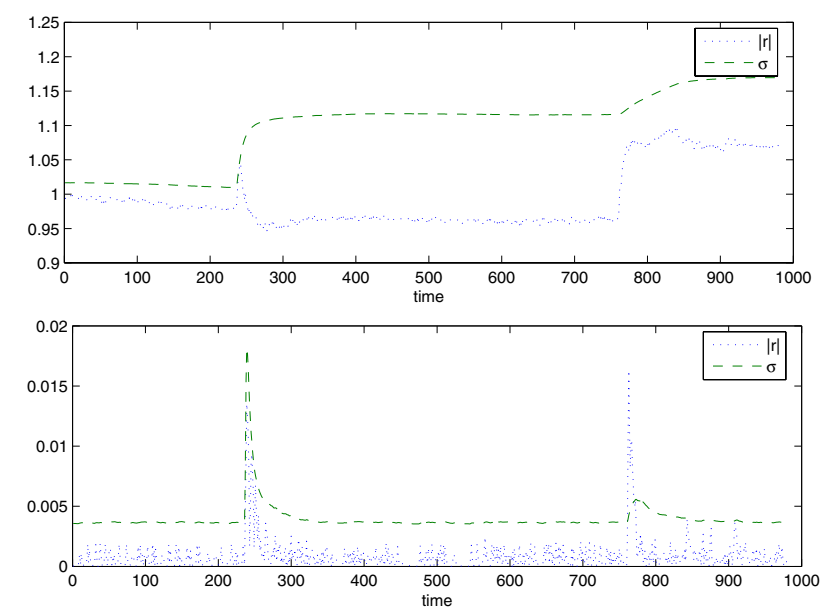

Fig. 4. An example where the method using an observer with integral action (lower figure) detects an error which the one without integral action (upper figure) does not detect.

is also presented which makes the method easy to use without any specific knowledge of the process except for the $\lambda$-tuning parameters and some upper bound of the disturbances.

In the future the design method for the threshold parameters should also include tuning of the upper bounds for measurement and process noise. It would also be of interest to make the threshold able to handle uncertainties in the time delay parameter.

\section{REFERENCES}

[1] Michael Bask Andreas Johansson and Torbjörn Norlander. Dynamic threshold generators for robust fault detection in linear systems with parameter uncertainty. Automatica, 42(7), 2006.

[2] Michael Bask and Andreas Johansson. Linear optimization of parameters in dynamic threshold generators. 16th IFAC World Congress in Prague, Czech Republic, 2005.

[3] Magnus Berndtsson and Andreas Johansson. Dynamic threshold generator for supervision of $\lambda$-tuned control loops. American Control Conference, Minneapolis, USA, 2006.

[4] Krister Forsman and Andreas Stattin. A new criterion for detecting oscillations in control loops. European control conference, Karlsruhe, Germany, 1999.

[5] P.M. Frank and X. Ding. Survey of robust residual generation and evaluation methods in observer-based fault detection systems. Journal of Process Control, 7(6):403-424, 1997.

[6] T. Hägglund. A control loop performance monitor. Control Engineering Practice, 3(11):1543-1551, 1995.

[7] Thomas J.Harris. Assessment of control loop performance. The Canadian Journal of Chemical Engineering, 67:856-861, 1989.

[8] Tina Miao and Dale E. Seborg. Automatic detection of excessively oscillatory feedback control loops. International Conference on Control Applications, Hawaii, USA, 1999.

[9] N.J. Sell. Process Control Fundamentals for the Pulp and Paper Industry. Tappi Press, 1995.

[10] Skogsindustriernas Teknik AB. Regleroptimering, ssg 5253 edition, 1997.

[11] N.F. Thornhill and T. Hägglund. Detection and diagnosis of oscillations in control loops. Control Engineering Practice, 5(10):1342-1354, 1997. 